\title{
Liquid Testing with Your Smartphone
}

\author{
Shichao Yue \\ Computer Science \& Artificial Intelligence Lab \\ Massachusetts Institute of Technology \\ Cambridge, Massachusetts, USA \\ scyue@mit.edu
}

\begin{abstract}
Surface tension is an important property of liquids. It has diverse uses such as testing water contamination, measuring alcohol concentration in drinks, and identifying the presence of protein in urine to detect the onset of kidney failure. Today, measurements of surface tension are done in a lab environment using costly instruments, making it hard to leverage this property in ubiquitous applications. In contrast, we show how to measure surface tension using only a smartphone. We introduce a new algorithm that uses the small waves on the liquid surface as a series of lenses that focus light and generate a characteristic pattern. We then use the phone camera to capture this pattern and measure the surface tension. Our approach is simple, accurate and available to anyone with a smartphone. Empirical evaluations show that our mobile app can detect water contamination and measure alcohol concentration. Furthermore, it can track protein concentration in the urine, providing an initial at-home test for proteinuria, a dangerous complication that can lead to kidney failure.
\end{abstract}

\section{CCS CONCEPTS}

- Human-centered computing $\rightarrow$ Smartphones; Mobile computing; Ubiquitous and mobile computing design and evaluation methods; • Applied computing $\rightarrow$ Physics.

\section{KEYWORDS}

Surface tension; liquid identification; mobile sensing; ubiquitous computing

\section{ACM Reference Format:}

Shichao Yue and Dina Katabi. 2019. Liquid Testing with Your Smartphone. In The 17th Annual International Conference on Mobile Systems, Applications, and Services (MobiSys '19), June 17-21, 2019, Seoul, Republic of Korea. ACM, New York, NY, USA, 12 pages. https://doi.org/10.1145/3307334.3326078

\section{INTRODUCTION}

Mobile computing has recently seen a surge in research on inexpensive methods for measuring liquid properties, and identifying liquid type [11, 18, 27, 35]. The developed methods can detect water contamination, and distinguish a variety of liquid types including

Permission to make digital or hard copies of all or part of this work for personal or classroom use is granted without fee provided that copies are not made or distributed for profit or commercial advantage and that copies bear this notice and the full citation on the first page. Copyrights for components of this work owned by others than the author(s) must be honored. Abstracting with credit is permitted. To copy otherwise, or republish, to post on servers or to redistribute to lists, requires prior specific permission and/or a fee. Request permissions from permissions@acm.org.

Mobisys' 19, Jun 2019, Seoul, South Korea

(C) 2019 Copyright held by the owner/author(s). Publication rights licensed to ACM. ACM ISBN 978-1-4503-6661-8/19/06 .. \$15.00

https://doi.org/10.1145/3307334.3326078

\author{
Dina Katabi \\ Computer Science \& Artificial Intelligence Lab \\ Massachusetts Institute of Technology \\ Cambridge, Massachusetts, USA \\ dk@mit.edu
}

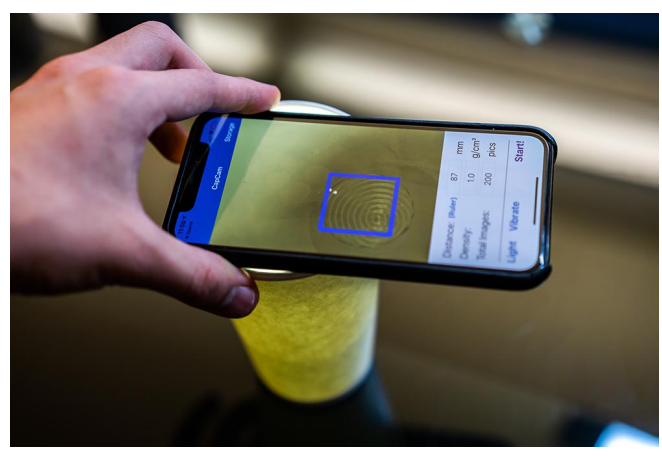

Figure 1: CapCam setup: The phone is placed on a paper cup. Capillary waves are generated by the vibro-motor inside the smartphone. Then with the flashlight, the camera of the phone can capture a bright-and-dark pattern, from which we can calculate the surface tension of the liquid.

water, milk, oil, and different alcohol concentrations. The goal of this line of research is to enable liquid testing outside the lab environment, and encourage ubiquitous applications. However, the proposed designs require a specialized setup (a robot [35], a special container [11], etc.), and use devices typically unavailable to the general population (e.g., UWB radios, or RFID readers). While they make an important step toward ubiquitous liquid testing, they are still difficult to use by lay users.

This paper asks whether it is possible to deliver such services to lay users without a specialized setup, and using only a device that almost everyone has: a smartphone. Answering this question is not simple. Typically, liquid testing is done by measuring a particular property, such as electric permittivity or optical absorption, and using the measurements to identify the liquid type and characteristics [11, 18, 27, 35, 41]. However, none of the properties used in past work can be measured with a smartphone. To address this problem, we explore an alternative liquid property, surface tension, and develop algorithms and system architectures that enable measuring surface tension using only a smart phone.

Surface tension characterizes the force that holds the surface molecules together, and minimizes the surface area. Measuring surface tension can reveal water contamination, and allow for distinguishing liquid types [7, 30]. Water has a relatively high surface tension [39], and when polluted with organic compounds such as bacteria, oil, petroleum or its derivatives, its surface tension decreases significantly $[7,30]$. Hence, one may use this property to detect water contamination. Further, lipids and proteins act as surfactants, i.e., they reduce surface tension. Thus, measurement of surface tension may be used to detect adulterated milk [2, 31, 37]. 
Alcohol also reduces surface tension, a property that can be leveraged to measure alcohol concentration [33]. Most interestingly, the ability to measure surface tension at home can enable early detection of medical problems. For example, low surface tension of urine may indicate the presence of excess protein, a dangerous complication in diabetes patients [12, 20,32]. Daily measurements of urine surface tension help detect diabetic nephropathy (the chronic loss of kidney functions), and monitor the effect of treatment [12].

Today, measurements of surface tension require a device called tensiometer, which typically costs thousands of dollars [15]. They are often conducted by dipping a platinum plate into the liquid, and carefully measuring the force required to pull it out. The process is complicated and requires professional training [15]. This high bar hampers the ubiquitous application of surface tension.

We introduce CapCam(Capillary Camera), the first mobile app that measures liquid surface tension using only a smartphone. To measure surface tension, the user places the smartphone on top of a light-weight container, like a paper cup, and activates the app, as in Fig. 1 . The phone vibrates and forces the container's wall to vibrate. The vibration generates capillary waves on the liquid surface, i.e., small waves whose wavelength characterizes the liquid's surface tension. Our app uses the flashlight camera to take a few photos of the liquid, which it processes to estimate the capillary wavelength and hence the surface tension. Our approach is accurate, simple, cheap, and accessible to any user with a smartphone.

Measuring capillary waves just using smartphones is quite challenging for two reasons. First, the waves are very shallow [4]; simply trying to image them at the liquid surface does not yield accurate results. Thus, instead of imaging the capillary waves directly, we image their reflections at the bottom of the container. We model the small waves on the surface as a series of convex and concave lenses. When illuminated with the flashlight on the phone, the lenses focus the light and create a pattern of bright and dark rings on the bottom of the container. The focusing effect of the small lenses makes this pattern much higher contrast than the waves on the surface, and hence easier to capture and characterize using a camera.

The second challenge stems from the camera rolling shutter. Phone cameras do not capture images in one snapshot; they capture a picture by sequentially scanning rows of photo diodes [22] Since the waves are moving, the sequentially scanning creates motion artifacts in the captured image. If uncorrected, these artifacts can lead to large measurement errors. CapCam addresses this problem by recognizing that the direction orthogonal to the scanning direction is not affected by motion artifacts; it processes the image to identify this orthogonal direction, and uses the line of pixels along that direction to measure capillary waves.

We have implemented CapCam on an iPhone X, and evaluated its performance by comparing its output against that of a high-end digital tensiometer. Our evaluation reveals the following findings:

- CapCam accurately measures liquid surface tension using only a smart phone. Specifically, CapCam has an absolute surface tension error of only $0.75 \mathrm{mN} / \mathrm{m}$. In comparison, an entry-level manual tensiometer [21] has a resolution of $0.5 \mathrm{mN} / \mathrm{m}$, costs several thousand dollars, and requires expert knowledge.
- CapCam has enough resolution to detect small differences in alcohol concentration of $0.5 \%$, whereas past work is limited to large differences of $20 \%$ for Nutrilyzer [27] and $25 \%$ for RFIQ [18]. CapCam's resolution allows it to easily detect water contamination and distinguish between clean water, water from puddles and water that was left exposed for a long period. CapCam also has enough sensitivity to track changes in protein levels in urine as they transition from healthy to dangerous levels, providing an initial at-home test of proteinuria.

Contributions: This paper makes the following contributions:

(1) It introduces CapCam, the first design that estimates liquid surface tension using only a smartphone, without any specialized hardware. CapCam is also the first mobile app that detects water contamination, measures alcohol concentration, and tracks changes in protein levels in urine.

(2) It presents multiple novel algorithms including: 1) an algorithm for inferring capillary wavelength by creating a characteristic pattern on the bottom of the container, and 2) an algorithm for characterizing the impact of rolling shutter on capillary waves and eliminating the resulting measurement errors.

(3) It provides an implementation and empirical evaluation that demonstrate the efficacy of the proposed design.

\section{RELATED WORK}

\subsection{Liquid Testing in Mobile and Ubiquitous Computing}

The topic of liquid testing has recently attracted a significant interest $[11,18,27,35,41]$. Most proposals try to infer electric permittivity [11, 35, 41], a property that characterizes how a liquid affects radio waves. For example, TagScan [35] and LiquID [11] measure the time delay and power attenuation incurred by an RF signal as it traverses the liquid of interest. Their approach requires a complex setup (a moving robot, or a particular container) and uses special radios not typically used by lay users. Further, since $\mathrm{RF}$ attenuation and phase are highly sensitive to liquid depth, these systems have to be carefully calibrated. As a result, these methods either exhibit relatively large errors ( $10 \%$ in LiquID [11]) or they avoid measuring exact values and resort to classifying the liquid as one of a few known types (as in TagScan [35]). There are also proposals that identify liquids using RF coupling, as in RFIQ [18], or $\mathrm{RF}$ reflections as in RadarCat [41]. They too use special radios (Soli, or RFID readers); further they rely on a classifier to distinguish a few liquid types or a few concentration levels, and do not generalize to unseen liquid types or concentration levels.

Some proposals rely on optical absorption. Specifically, when shining intensity-modulated light on a liquid, different liquids produce uniquely different sound spectra. These solutions require custom hardware and have a relatively limited resolution. For example, by analyzing the received spectra, Nutrilyzer [27] can predict alcohol concentration level with a limited resolution $(20 \%)$.

CapCam is inspired by the above work, but focuses on liquid testing using a smartphone, a device that almost every user has. 


\subsection{Measuring Surface Tension}

Today, surface tension is measured in the lab using an expensive device called tensiometer [40]. Tensiometers differ in the physical property they use for their measurements:

- Force: Most tensiometers measure the force that brings the surface molecules together. They operate by submerging a platinum probe (like Du Noúy Ring [14] or Wilhelmy plate [13]) in the liquid, and precisely measuring the force required to pull the probe out.

- Pendant drop: This method measures surface tension by analyzing the curved shape of a drop of liquid. [5, 19]. It requires extreme cleanliness, and a tight control over the drop size, and the measurement procedure [5, 13].

- Contact angle: This method measures surface tension by measuring the contact angle between a drop of liquid and some solid surface [42]. However, the equilibrium contact angle can vary within a range and depends on the drop size. Thus, this method requires a sophisticated measurement procedure [28, 42].

- Capillary waves: This method measures surface tension by vibrating the liquid and measuring the capillary waves on the surface. Since the amplitude of the capillary waves is extremely small, researchers usually measure the wavelength using a technique called optical diffusion [3, 24, 43]. It requires shining a directional laser on the surface and analyzing the diffraction pattern on a receiving screen.

Although these methods can achieve high accuracy, expensive instruments and sophisticated measurement procedures prevent their ubiquitous use. In comparison, CapCam extends the model based on capillary waves to allow a non-expert user to measure surface tension using only a smart phone.

While some past work has attempted to measure surface tension using a smart phone, all past proposals require additional complex hardware, and none is able to complete the measurements with only a smartphone $[8,9,17,36]$. Specifically, the work in Wei et al. [36] uses a cell phone camera to capture images of capillary waves. However, it ignores the rolling shutter effect, which leads to poor performance. Further, it requires custom hardware where a large container is elevated between two stands while projecting light from the bottom, a signal generator that excites the liquid, and a paper screen held on top of the surface. Both Goy et al. [17] and Chen et al. [9] are based on the Pendant Drop method. They use cellphone cameras to capture images of a drop of liquid. The method requires specialized equipment to exercise tight control of the drop's size and shape. It also requires extreme cleanliness and a complex measurement procedures making it hard to conduct by non-experts. Chen et al. [8] is based on the Contact Angle method. As in the previous methods, it leverages a phone camera for analysis but ignores the rolling shutter, and requires custom hardware to control the size of the drop and its contact angle as it touches a special solid surface.

CapCam builds on the above work. However, unlike these methods, CapCam can measure surface tension simply using a smartphone app without any special hardware. Further, it can be operated by an average user without any training.

\section{CAPILLARY WAVES PRIMER}

In this section, we provide a primer on capillary waves and their relation to surface tension. For waves propagating on a fluid surface, gravity $(g)$ and surface tension $(\gamma)$ are the forces that make the liquid restore its flat surface. Those forces control the dispersion relation of the wave, which relates its wavelength $(\lambda)$ to the vibration frequency $(f)$, and liquid density $(\rho)$, and can be expressed by the following equation (see Chapter. 16 of Blandford and Thorne [6] for a detailed derivation):

$$
(2 \pi f)^{2}=g(2 \pi / \lambda)+\frac{\gamma}{\rho}(2 \pi / \lambda)^{3} .
$$

When the waves are large, like those observed on the surface of a lake, they are dominated by the gravity term $g$, and referred to as gravity waves. When the waves are small, the effect of gravity is negligible, and the waves are dominated by surface tension $\gamma$; they are called capillary waves.

We can re-order the terms in the above equation to measure surface tension as follows:

$$
\gamma=\frac{(2 \pi f)^{2}-g(2 \pi / \lambda)}{(2 \pi / \lambda)^{3}} \rho .
$$

This equation provides a procedure for measuring surface tension using capillary waves. Specifically, we can use a vibration source to generate capillary waves on the liquid's surface. Knowing the vibration frequency $f$, we can substitute the gravity term and the liquid density from the corresponding data sheets. ${ }^{1}$ Hence, all we need is to measure the wavelength $\lambda$ in order to measure the surface tension.

The challenge however is that capillary waves are very shallow -i.e., the displacement they cause in the liquid surface is one to a few microns. This is about one tenth of the average thickness of a human hair. Thus, such waves are invisible and their measurement typically requires dedicated laboratory equipment [3, 24, 36, 43] with a complicated setup and procedure. In the rest of this paper, we describe CapCam, a novel design that can measure capillary wavelength using only a smartphone.

\section{CAPCAM DESIGN}

CapCam measures surface tension using a phone's camera, flashlight, and vibro-motor. The process is very simple. The user places the smartphone on top of the container (e.g., a cup) and activates the CapCam app, as in Fig. 1. CapCam uses the phone's vibro-motor to generate capillary waves on the liquid surface, then takes a few photos of the vibration pattern using the phone flashlight camera. It uses a series of algorithms to process these photos to infer the capillary wavelength and hence the surface tension.

CapCam's inference algorithm addresses the challenge of measuring very shallow capillary waves, and makes it possible to measure the wavelength with only a smartphone. In this section, we describe the model and analysis underlying CapCam's wavelength inference algorithm. We describe how we deal with phone hardware (e.g., rolling shutter) in the next section.

\footnotetext{
${ }^{1}$ Liquid density is a constant given temperature and pressure. For most applications, one can assume room temperature and atmosphere pressure. If the measurements are conducted at unusually high/low temperatures or very high elevation, one should substitute the corresponding liquid density from the liquid's data sheets.
} 


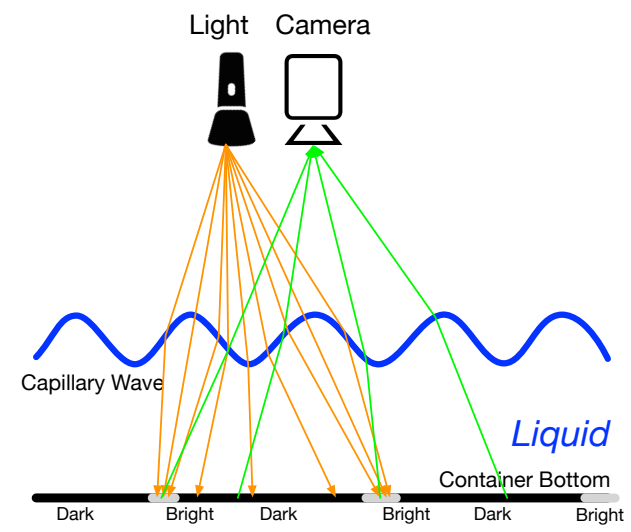

Figure 2: An cross-section illustration of the setup. Because of refraction, light rays are focused/diverged by the crests/troughs of the waves, resulting in a series of dark and bright rings on the bottom.

\subsection{CapCam's Wavelength Inference Algorithm}

We model the crests and troughs of capillary waves as a series of convex and concave lenses. By shining a flashlight on the waves from the top, we can create a visible pattern that reflects off the bottom of the container. Specifically, and as shown in Fig. 2, when the light goes through a wave crest, it is focused into a small area on the bottom of the container. On the other hand, when the light goes through a wave trough, it diverges causing a dark region on the bottom of the container. This results in a series of bright and dark rings on the bottom of the container.

If we try to capture this pattern using a smartphone, the camera would see only the reflections that exit the liquid surface and propagate through air towards the camera. However, due to reciprocity [25], light reflected from the bottom towards the surface experiences the opposite effects of light entering the surface from air -i.e., light rays from the bright rings will diverge and light rays from the dark rings will converge. Hence, if the camera is exactly at the same position as the flashlight, it will not see any pattern. However the camera is never at the exact same location as the flashlight. Hence the light rays that it receives do not trace the exact path of the incoming light. As a result, the focus and divergence effects that the light experienced while traversing the surface from air to liquid do not get cancelled as it traverses the surface from liquid to air. Hence, the pattern continues to be visible to the camera albeit at lower contrast.

After we take an image of the pattern at the bottom of the container, we measure the distance between two consecutive bright rings in terms of pixels, denoted as $p$. We need to convert $p$ from pixel-based distance to real distance. Of course, this depends on the distance between the camera and the bottom of the container, which we denote as $d+h$, where $d$ is the distance between the camera and the surface of the liquid, and $h$ is the depth of the liquid. Let us use $r_{d+h}$ to refer to the resolution of the camera for objects at distance $d+h$. Then, we can convert the distance between consecutive bright

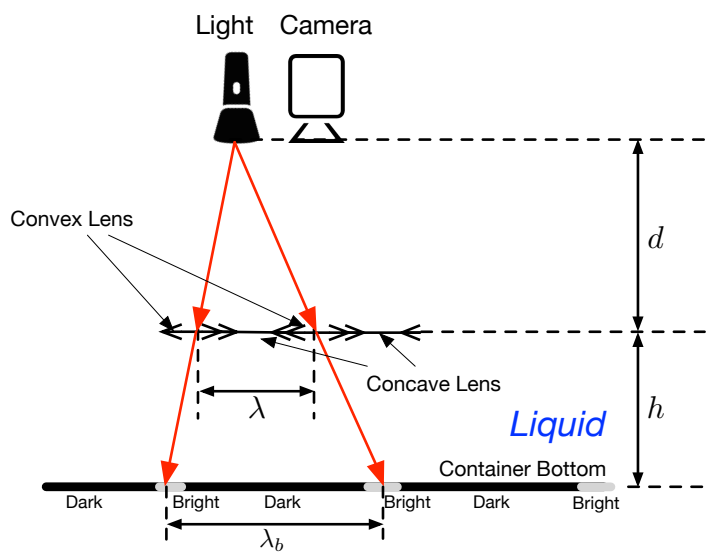

Figure 3: An illustration of the wavelength calculation. We plot only the rays that pass through the center of the crests of the waves, which do not bend.

rings in the image, $p$, into real distance, $\lambda_{b}$, as follows:

$$
\lambda_{b}=p / r_{d+h}
$$

Next, we want to use the interval between two bright rings to estimate the capillary wavelength. To calculate the relationship between the two, we approximate the surface waves as perfect lenses; when the incident ray goes through the center of a lens, its direction is unchanged, as shown in Fig. 3. Thus, the relation between the capillary wavelength $\lambda$ and the interval between two bright rings, $\lambda_{p}$ is:

$$
\lambda=(d / d+h) \cdot \lambda_{b}
$$

Combining the two equations, we have:

$$
\lambda=\frac{d}{d+h} \cdot \lambda_{b}=\frac{d}{d+h} \cdot \frac{p}{r_{d+h}} .
$$

Since camera resolution is inversely-proportional to distance from the imaged object, we have:

$$
r_{1} * d_{1}=r_{2} * d_{2}
$$

Therefore, we can rewrite Equ. 5 as follows:

$$
\lambda=\frac{p}{r_{d+h} *(d+h) / d}=\frac{p}{r_{d}},
$$

where $p$ is the number of pixels between two consecutive bright rings, and $r_{d}$ is the camera resolution for objects at distance $d$, i.e., at the liquid surface.

Equ. 7 and the model underlying it provide us with an algorithm to compute the capillary wavelength. They also show that, we only need to measure the distance between the camera and the liquid surface, and use the corresponding resolution to convert the inter-ring pixels in the image to the actual capillary wavelength.

Note that, it is enough to calibrate the camera resolution at one default distance based on Equ. 6. The resolution $r$ at distance $d$ can be computed as

$$
r=\left(r_{0} \times d_{0}\right) / d
$$

where $r_{0}$ is the resolution at the default distance $d_{0}$ 


\subsection{Illustrative Simulation}

To provide a better visual understanding of CapCam's algorithm, we build a simulator based on ray tracing. The surface of the liquid and the bottom of the container are discretized into dense pixels. Our simulation can be divided into two phases. First, the light source emits light rays onto each pixel on the surface, and for each light ray, we calculate its location of arrival at the bottom of the container. Then the intensity of each bottom pixel is set to the total number of rays falling on that pixel. In the second phase, we densely sample locations inside each pixel in the camera, and trace the light rays that fall on the camera to their origin on the bottom of the container. This allows us to obtain the set of pixels on the bottom of the container that are reachable from that camera pixel The intensity at each camera pixel is set to the sum of the intensity of all reachable bottom pixels.

We show the results of a simulated experiment in Fig. 4. By comparing Fig. $4 \mathrm{~d}$ with Fig. $4 \mathrm{e}$, we can see that the peaks of the pattern on the bottom of the container are much sharper than the crests of the capillary waves at the surface. This is because the crests of the surface waves focus light into a series of much narrower bright rings at the bottom of the container, therefore increasing the contrast. Also by comparing Fig. $4 \mathrm{f}$ with Fig. 4 e, we can see that troughs of the pattern captured by the camera are distorted and the peaks are shifted to the left. This is because the slight separation between the camera and the light source which causes the camera to see the pattern from its own angle. But still, we can use the imaged pattern to calculate the wavelength accurately using Equ. 7. In this simulation, the camera has a resolution of 40 pixel-per-millimeter at the surface distance, and the wavelength is $3 \mathrm{~mm}$. At these values, and assuming the liquid is water, the wavelength is exactly 120 pixels.

\section{DEALING WITH SYSTEM CHALLENGES}

The algorithm in section 4 measures the surface tension using the following: a high frequency vibro-motor that can be used to excite capillary waves, a light source to provide illumination, and a camera to capture the resulting pattern. Thankfully, a smartphone includes all three components.

Thus, in principle, we can measure the wavelength in the image captured by the phone, and convert from pixel-based distance into real distance using Equ. 7, which gives us the surface tension. But in reality, we face several challenges due to the reality of phone hardware. Particularly, there are two main factors that prevent us from measuring the wavelength accurately: the camera's rolling shutter and the instability of the phone's vibration frequency.

\subsection{Rolling Shutter Effect}

As explained earlier, the wavelength is the distance between two consecutive bright rings. Since the rings are concentrated (see Fig. 4c), we can pick a particular direction along the radius of the rings and use it to measure the distance between consecutive bright rings, which would then yield the wavelength. Unfortunately, it is not that simple. In reality, not all directions give the correct wavelength. Fig. 5 shows an example image of the ring pattern due to capillary waves. The figure shows three radial directions along and the corresponding wavelength in number of pixels. The figure shows that the average number of pixels between two consecutive bright rings along these three directions is 109,111 , and 115 . The differences between these estimates of the wavelength cause a significant difference in the resulting estimate of surface tension. In fact a difference of two pixels leads to an error of $4 \mathrm{mN} / \mathrm{m}$ in surface tension, which is more than $5 \%$ of the water's surface tension $\gamma=72 \mathrm{mN} / \mathrm{m}$. This means that we have to figure out which of these radial directions yields the correct estimate of wavelength, and use only that direction.

In order to pick the correct radial direction for our estimation, we first need to understand why different directions yield different distances between the bright rings, i.e., different wavelengths. The reason is the rolling shutter. Rolling shutter refers to that a camera captures a picture by sequentially scanning rows of photo diodes [22]. Therefore pixels are not recorded at exactly the same instant, and since the wave is traveling rapidly, this will result in a Doppler Effect.

In the example in Fig. 5, the camera is scanning from right to left, and the wave is propagating from the edge of the cup to the center. The propagation direction and the scanning direction are aligned for the radius on the right (in the figure), and are opposite for the radius on the left. Thus, the measured wavelength is shorter along the left radius and longer along the right radius. As for the radius in the middle, the propagation direction of the wave is perpendicular to the camera scanning direction, hence the measurement is not affected by the rolling shutter.

Therefore for accurate estimation, we want to measure the wavelength along the radius perpendicular to the camera's scanning direction (the green line in Fig. 5). Given that the camera scan images horizontally, the correct radius is along the vertical direction in the image. We can ask the user to manually pick the vertical radius. However it is preferable to do it automatically to reduce user overhead and any potential errors. To do so, we search for the vertical line in the image that maximizes symmetry. Specifically, since the line we are looking for is a radius of the concentrated rings, the pattern on its left should be similar to the pattern on the right (i.e., the intensity of the few pixels to the left of the line is similar to the intensity of the few pixels to the right of the line). Thus, we only need to find a vertical line whose neighbor pixels are symmetric with respect to itself. This is similar to detecting reflection symmetry in images and can be solved by convolving with a wavelet filter [10]. Specifically, we design a wavelet filter (shown in Fig. 6) that is -1 on the left side and +1 on the right side. When convoluting with a image, result will be close to zero only when there is reflectional symmetry in the reception field of the filter.

The reflection symmetry is only observable when the reception field of the filter is large enough, e.g. at the scale of one wavelength, which is about a hundred pixels. To increase the reception field of the filter, instead of use a big filter which is expensive, we can down-scale the image to speed up the convolution process. Then for each column in the convolved image, we calculate the sum of the absolute values of all of its pixels. Because of the property of the filter, the result in the ideal column should have the lowest value. Therefore, we select the column with minimum sum as the ideal location to measure the wavelength. The algorithm is visualized 


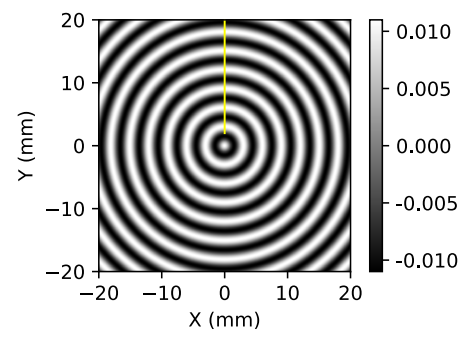

(a) Wave

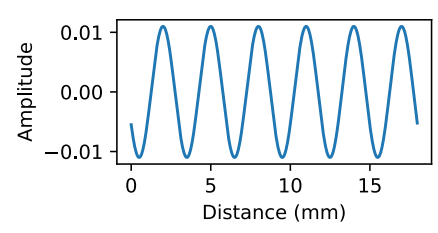

(d) Cross section of Wave

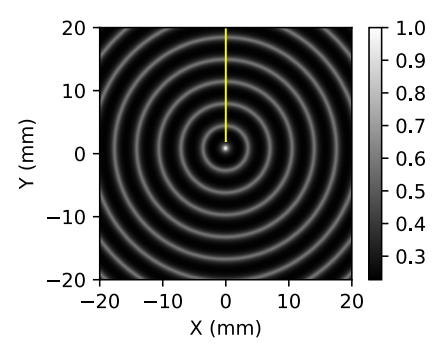

(b) Bottom Pattern

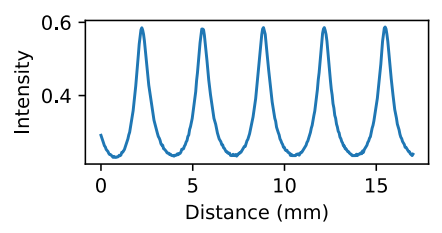

(e) Cross section of Pattern

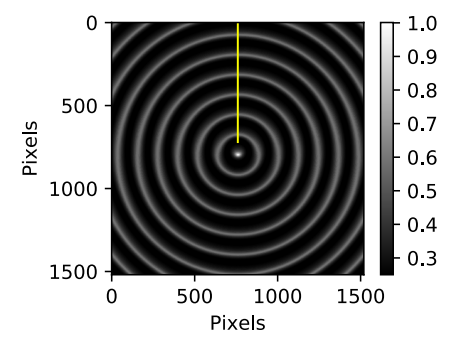

(c) Captured Image

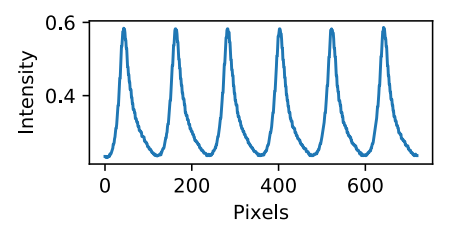

(f) Cross section of Image

Figure 4: A simulated experiment showing the relation between the waves on the surface, the pattern on the bottom of the container and the captured image. Figures on the second row show the pixel value along the yellow line in the corresponding first row figure. Comparing $4 \mathrm{~d}$ with $4 \mathrm{e}$, we see that the crests of the pattern on the bottom are both sharper and higher value than the crests of the waves on the surface. This is due to the focusing effect on the lenses. Also note that in $4 \mathrm{f}$, the crests are shifted to the left; this is because the camera and the light are not co-located.

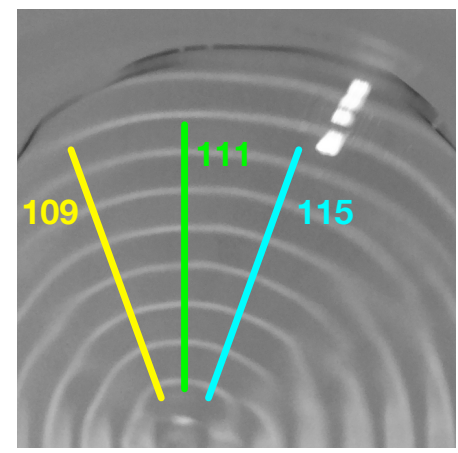

Figure 5: A sample image taken by a smartphone. The three lines represent three radii with different directions. Numbers besides the lines are the corresponding wavelength measured in number of pixels, and they are different from each other. This suggests that picking a wrong radii may result in a significant error.

in Fig. 7. Note that, in the implementation, we also average across multiple images to improve robustness of the algorithm.

This algorithm can locate the vertical radius accurately. Further, since it is based on very simple operations, it is highly efficient and can run on an ordinary smartphone.

\subsection{Unstable Vibration Frequency}

When vibrating, the frequency of the vibro-motor on the phone is not always constant; it shows small perturbation around the center frequency. Further, because of API restrictions, smartphones are forbidden to vibrate continuously. Instead, they have to follow

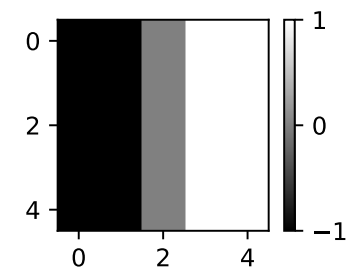

Figure 6: The wavelet filter for detecting local reflectional symmetry. Values on left side are all -1 , and +1 on the other side. And values in the middle column is 0 . The convolution result will be zero when there is reflectional symmetry.

a vibrate-stop-vibrate pattern. Therefore the vibro-motor need to constantly accelerate and decelerate, results in an unstable vibration frequency. The randomness in the vibration frequency affects the capillary waves, making the wavelength measured from a single image unreliable.

To deal with this issue we use a large number of images in our estimation. Fortunately, smartphones are able to capture images at a very high frame rate (e.g. iPhone's burst mode). Thus, we can obtain hundreds of images within a few seconds. Since the location of the phone is not changed during this short period, we can use all of those images to obtain a more robust location for computing the wavelength using previous method. Then for each image, we extract the pixel value on the column we have selected, and apply a peak detection algorithm to extract all the peaks, and calculate the interval between every two peaks. To combat with the unstable frequency, we create a histogram of the all wavelength estimates. Noticing that, because of the vibrate-stop-vibrate pattern that the 


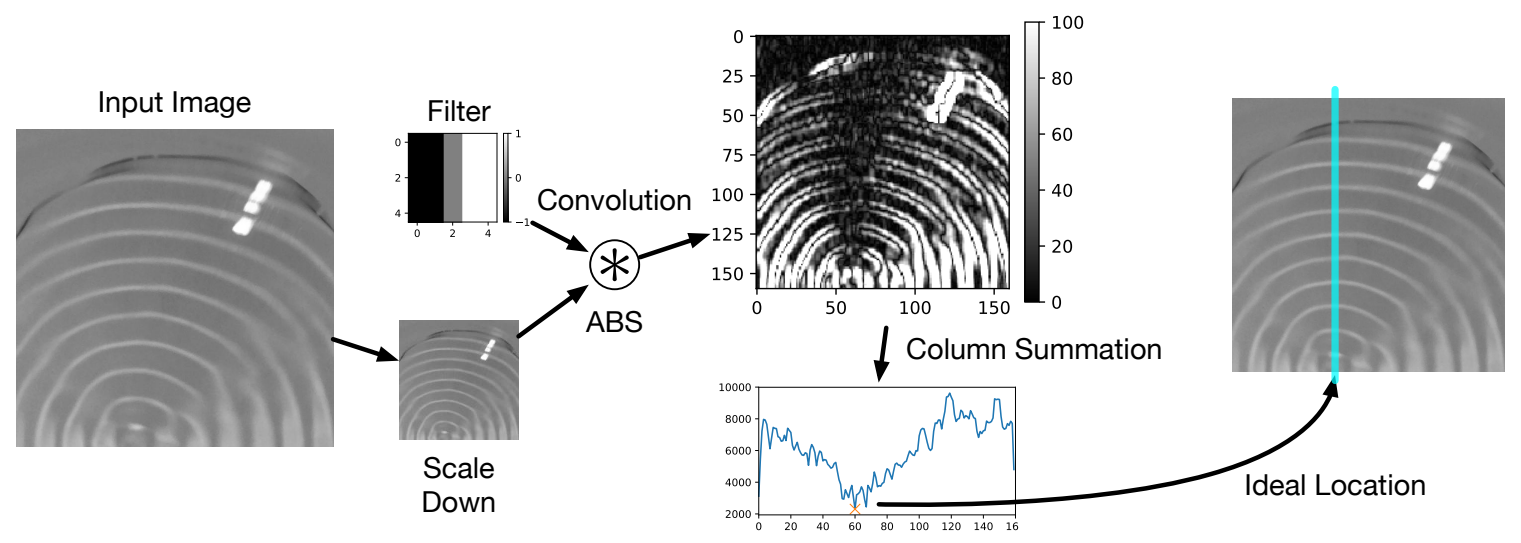

Figure 7: The algorithm for identifying the ideal location along which to measure the wavelength. We first scale down the image, and convolute it with a filter for detecting local symmetry. Then calculate the sum of the absolute values for each column. Then the column with lowest sum is the location where its wavelength is not affected by the rolling shutter effect.

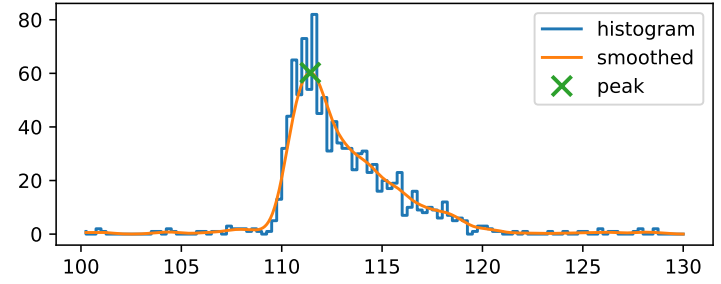

Figure 8: A sample histogram of estimated wavelengths with 200 images.

motor follows, a simple average across all the wavelengths will result in an estimation bias. Instead, we smooth the histogram with a Gaussian filter, then pick the location of the maxima to be the estimated wavelength. A histogr am generated with 200 images is shown in Fig. 8

\section{USER INTERFACE AND IMPLEMENTATION}

We implement CapCam as a standalone iOS App using the Swift programming language. To speedup processing, we use Apple's Accelerate Framework[1] for SIMD operations like convolution and summation. This yields over 1000x speedup compared to a naive Swift implementation.

Two main user interfaces of CapCam are shown in Fig. 9. The UI on the left (Fig. 9a) is the configuration interface. On this interface, we introduce each component from top to the bottom. The first component is the preview of the camera. On the preview, there is a blue frame showing the area where we run our analysis algorithm. By cropping the image, we are able to achieve a much faster speed without sacrificing accuracy. Next there are three text fields. The first text field is for the user to input the distance between the surface and the camera. The app includes a ruler to assist the user in measuring the distance directly with the phone. The second text field is for the density, and its default value is 1.0. The third text field is for the user to specify the number of captured images. Having more images will increase the accuracy, yet will take a longer time.

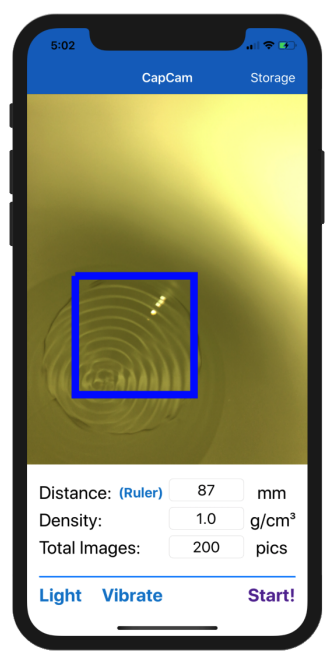

(a) Configuration

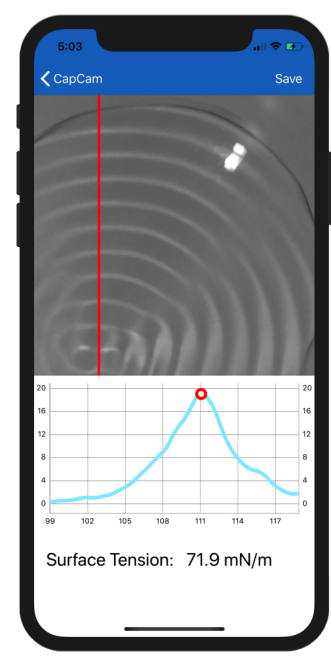

(b) Analysis
Figure 9: CapCam's User Interface

Then there is a progress bar indicating the progress of the analysis process. Finally, there are three buttons on the bottom. "Light" and "Vibrate" are the controls for the flashlight and the vibro-motor, and the "Start!" button is for starting the analysis process.

After the analysis finishes, the app shows the analysis interface, which is the UI on the right(Fig. 9b). One sample captured image is shown on the top half, with the automatically detected estimation radius highlighted. The histogram of the wavelength is plotted beneath the sample image. Finally, the estimate of the surface tension is printed out at the bottom.

\section{EVALUATION}

In this section we evaluate the performance of CapCam and its ability to deliver interesting applications to the user. 


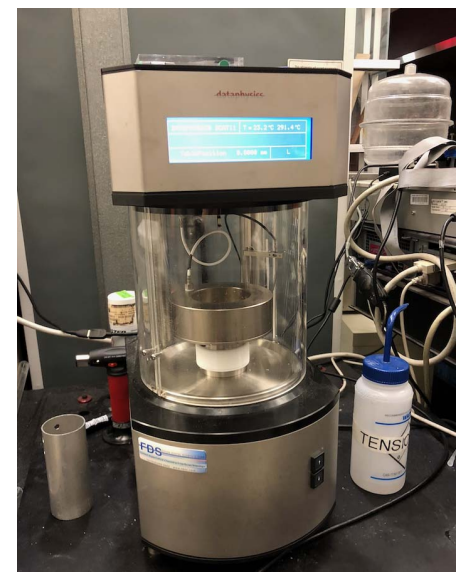

Figure 10: Digital tensiometer with a resolution of $0.1 \mathrm{mN} / \mathrm{m}$ for measuring ground truth of surface tension (Model: Dataphysics DCAT 11 [15])

\subsection{Ground Truth}

In all experiments, the ground truth of the surface tension is obtained by using an advanced digital force tensiometer (Dataphysics DCAT 11 [15]), shown in Fig. 10. It measures the surface tension using the Wilhelmy plate method, and can provide a resolution of $0.1 \mathrm{mN} / \mathrm{m}(0.1$ millinewton per meter).

\subsection{Experiment Setup}

We install CapCam on an iPhone X. Unless specified otherwise, we use a standard paper cup as a testing container, and set the liquid depth to $45 \mathrm{~mm}$. We place the phone on top of the cup, as shown in Fig. 1. We hold the phone with our hand as shown in the figure to ensure it stays still while take the images, and does not move due to vibrations. The cup has a height of $132 \mathrm{~mm}$. The camera on the iPhone is measured to have a resolution of 39.5 pixels per millimeter at a distance of $87 \mathrm{~mm}$, and the vibration frequency of the iPhone is centered at $144.5 \mathrm{~Hz}$. For each measurement, CapCam continuously takes 200 images. On average, each surface tension measurement takes 8 seconds. For each liquid sample, we repeat the measurement 5 times and compute the average and standard deviation. $^{2}$

\subsection{Detecting Water Contamination}

Water has a relatively high surface tension [39], and when polluted with organic compounds such as petroleum, bacteria, pesticides, oil or its derivatives, water surface tension decreases significantly [7, 30]. In this section, we empirically evaluate the effectiveness of CapCam at detecting changes in surface tension due to such contamination. While not all sources of water contamination change surface tension (e.g., metal contamination), our approach covers a large and important class of contaminants. Such bacterial and organic contamination is common anywhere unsanitary conditions are present. Further, people who live downstream from factories are at greater risk of contamination from petroleum and

${ }^{2}$ A demo video of our experiments is available at http://people.csail.mit.edu/scyue/ projects/capcam/

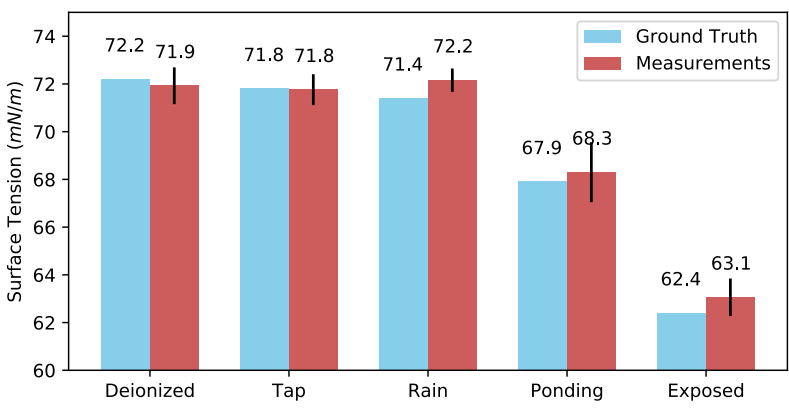

Figure 11: Water Contamination Detection. Both tap water and rain water have a surface tension close to deionized water. In contrast, pond water and exposed water have lower surface tension values due to contamination.

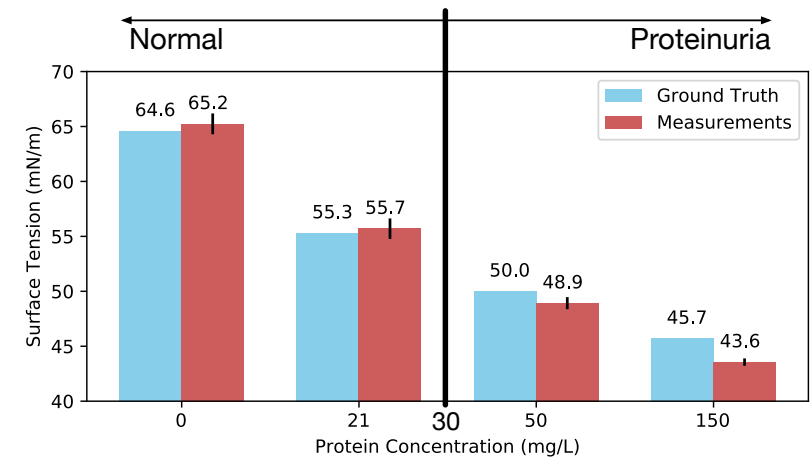

Figure 12: Urine surface tension test with different protein concentration levels. For a healthy person, protein concentration level in urine should be less than $30 \mathrm{mg} / \mathrm{L}$ [16], and the greater the concentration, the higher the risk.

organic waste, and people in farming communities are at risk for contamination from agriculture waste [26].

We compare 5 different water sources: 1 . deionized (i.e., pure) water, 2. tap water, 3. rain water, 4 . pond water from a tree pit, 5. water left exposed for a week. Fig. 11 plots the surface tension of the above water sources. The figure reveals two findings. First, by comparing the blue and red bars in the figure, we see that CapCam's measurements of surface tension match those from the tensiometer. This shows CapCam's accuracy. Second, the figure shows that both tap water and rain water have a surface tension similar to deionized water, which indicates that these sources of water are not polluted. On the other hand, pond water and exposed water have much lower surface tension, meaning that they contains chemicals that can decrease surface tension, which is a sign of contamination.

\subsection{Tracking Protein in Urine}

People who have diabetes or high blood pressure are vulnerable to kidney disease. When the kidney is damaged, it starts leaking substrates into urine that are not supposed to be present. Many of these substrates reduce urine surface tension. In particular, microalbuminuria is a complication in diabetic patients, where the kidney starts leaking albumin into urine [23, 34]. We would like CapCam 


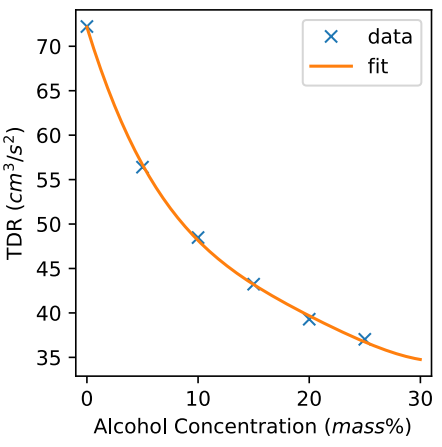

Figure 13: TDR as a function of ethanol concentration. Data points are obtained from Vazquez et al. [33] and Speight et al. [29]. We use a polynomial fit to obtain a continuous function between the concentration level and the TDR.

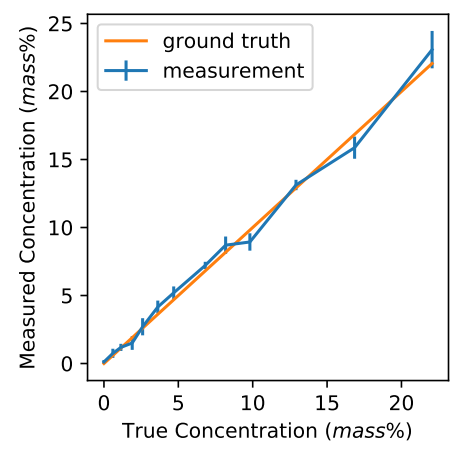

Figure 14: Alcohol concentration as measure by CapCam in blue and ground truth in orange.

to help diabetic patients by providing them with an easy way to regularly test the level of albumin in urine. If the level increases beyond a safe threshold, the patient can contact her doctor for more intensive tests.

We empirically test CapCam's effectiveness at delivering this application. We add different levels of egg albumin ${ }^{3}$ to a sample of healthy human urine, and measure urine surface tension for different albumin concentration.

Fig. 12 shows urine surface tension as a function of albumin concentration. Note that a protein level of over $30 \mathrm{mg} / \mathrm{L}$ is the threshold at which the patient has microalbuminuria [16]. The results in the figure indicate that our system can track changes in urine surface tension with increased albumin concentration in a manner comparable to a professional tensiometer. Further, it can detect when the protein concentration becomes dangerous. This means a patient can track the progress of the disease in the home using her smartphone.

\subsection{Measuring Alcohol Concentration Level}

We test the performance of our system on a series of ethanol solutions with different concentration levels. This is a complex scenario since an ethanol solution is a mixture of two liquids: water and

${ }^{3}$ Egg albumin and human serum albumin both belong to albumin, a family of globular proteins, and sharing similar physical properties [38].

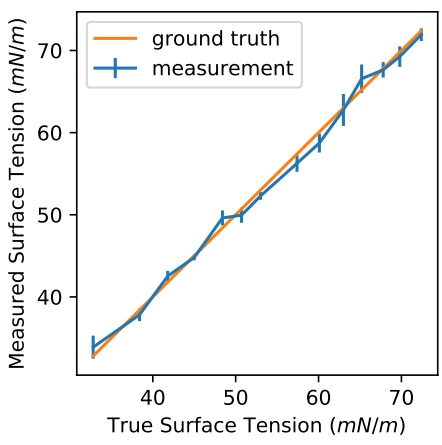

Figure 15: CapCam's resolution: differences between true and measured surface tension show that CapCam has an average error of $0.75 \mathrm{mN} / \mathrm{m}$

ethanol. The density of the mixture $\rho$ in Equ. 2, depends on the ratio of ethanol to water in the mixture. Thus, we are facing a chickenand-egg problem: we want to measure surface tension to estimate alcohol concentration; however we need the alcohol concentration to estimate $\rho$, which we need for our measurements of surface tension.

To address this issue, we leverage the fact that both the surface tension and the density of the ethanol mixture are functions of its concentration level. Further, they are known functions available in data sheets [29, 33]. Given both functions, we can compute for each given ethanol concentration level, its surface tension and its density, and take the ratio of the two, denoted as the surface tension to density ratio (TDR). We plot this ratio in Fig. 13. The curve in this figure allow us to compute alcohol concentration given the TDR.

We can then re-write Equ. 2, so that we can estimate the TDR, i.e., $\gamma / \rho$, as opposed to surface tension $\gamma$ :

$$
T D R=\frac{\gamma}{\rho}=\frac{(2 \pi f)^{2}-g(2 \pi / \lambda)}{(2 \pi / \lambda)^{3}} .
$$

Notice that the left hand side of this equation is the TDR, and we can compute it by substituting for the vibration frequency, gravity, and the wavelength of the capillary waves, which we can compute as before. Therefore we can first measure the TDR of the alcohol using CapCam, then convert the TDR into the concentration level based on the relation in Fig. 13.

The measurement result for ethanol concentration level is plotted in Fig. 14 . The absolute error is only $0.51 \%$. This implies that our system is accurate enough to distinguish German Riesling from Australian Riesling, and Portuguese Rose from French rosés.

\subsection{CapCam's Resolution}

Next we are interested in understanding CapCam's resolution, i.e., the expected error in its measurements of surface tension. To estimate this value, we leverage the results from the alcohol concentration experiment. Specifically, for any alcohol concentration level, there are datasheets that report the surface tension and density [33]. By substituting the density $\rho$ in the TDR equation above, we compute CapCam's estimate of surface tension, $\gamma$, which we can compare against the true surface tension in the datasheet. The 


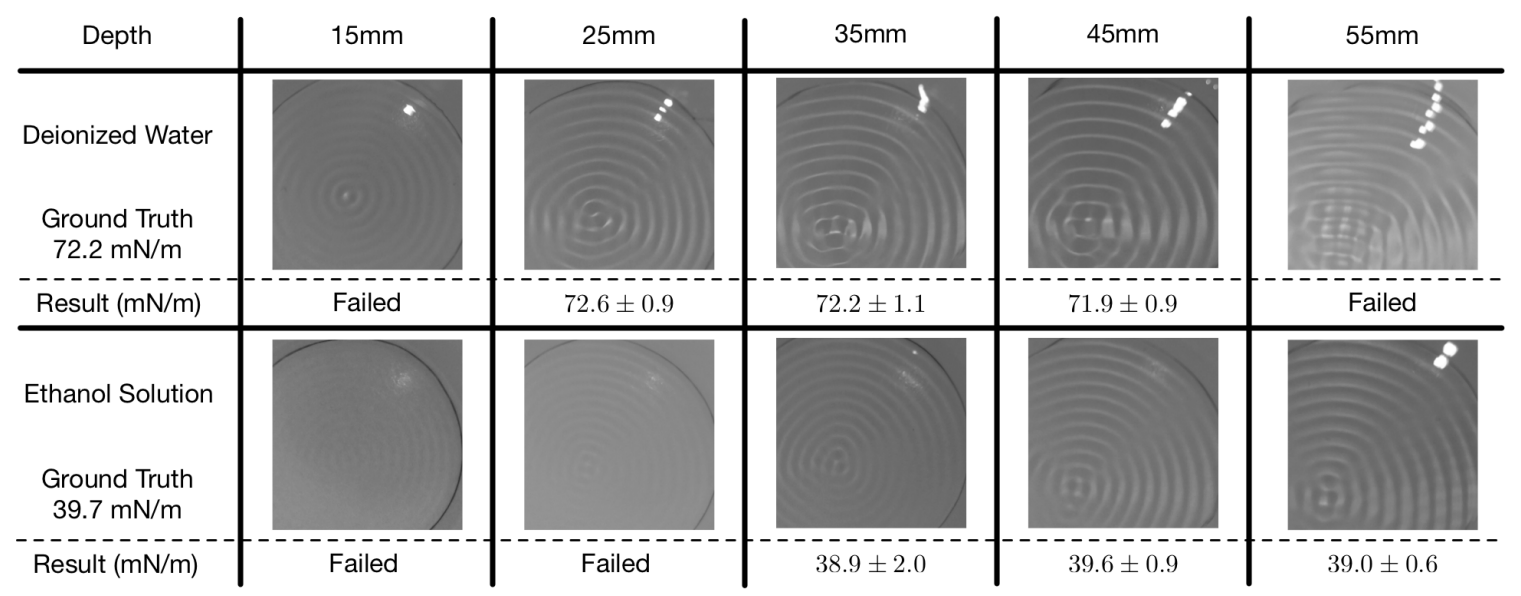

Figure 16: The impact of liquid depth on the quality of the captured image. When the focal length of the waves does not match the depth of the liquid, the bottom of the container may be out of focus. However, as the results indicates, even when the mismatch between the depth and the focal length is about $36 \%$ to $40 \%$ (20mm out of $55 \mathrm{~mm}$ for oil and $45 \mathrm{~mm}$ for water), our system can still measure the surface tension robustly.

measurements are plotted in Fig. 15. On average, CapCam has a surface tension error of only $0.75 \mathrm{mN} / \mathrm{m}$, for surface tension in the range from $33 \mathrm{mN} / \mathrm{m}$ to $72 \mathrm{mN} / \mathrm{m}$. In comparison, an entry-level manual tensiometer [21] a resolution of $0.5 \mathrm{mN} / \mathrm{m}$, and it still costs thousands of dollars and requires complicated procedure.

\subsection{Impact of Liquid Depth}

As explained in Sec. 4, we can capture a pattern of bright and dark rings because the capillary waves on the surface act like a series of convex and concave lenses. But every lens has a focal length. When the focal length of the waves does not match the depth of the liquid, the bottom of the container (i.e., the ring pattern) will be out of focus. Thus, in this experiment, we evaluate the sensitivity of our system to the choice of liquid depth. To do so, we use two types of liquids that vary significantly in their surface tension: deionized water $(\gamma=72.2 \mathrm{mN} / \mathrm{m})$ and ethanol solution $(\gamma=39.7 \mathrm{mN} / \mathrm{m})$.

We generate capillary waves and check when the surface tension estimated by CapCam matches the correct value. We repeat the experiment for 5 different depths: $15 \mathrm{~mm} / 25 \mathrm{~mm} / 35 \mathrm{~mm} / 45 \mathrm{~mm} /$ $55 \mathrm{~mm}$. The measurement results are shown in Fig. 16.

The figure shows that when the depth of the water ranges from $25 \mathrm{~mm}$ to $45 \mathrm{~mm}$, our system provides accurate measurements. The depth suitable for measuring the ethanol solution is larger, and spans the range from $35 \mathrm{~mm}$ to $55 \mathrm{~mm}$. This is because ethanol has a lower surface tension than water, hence the capillary wave on its surface have a smaller amplitude, and focus light at a larger depth Overall, the results in the figure show that even when the depth and the focal length are mismatched by $36 \%$ to $40 \%$ (20mm out of $55 \mathrm{~m}$ for oil and $45 \mathrm{~mm}$ for water), our system can still measure the surface tension robustly.

The figure also shows that when the depth is far too small or too large, the ring pattern is out of focus and is too blurred to allow for accurate measurements. However, our system can indicate to the user when the pattern is too blurred for accurate measurements. Such scenarios typically generate an abnormal range for surface

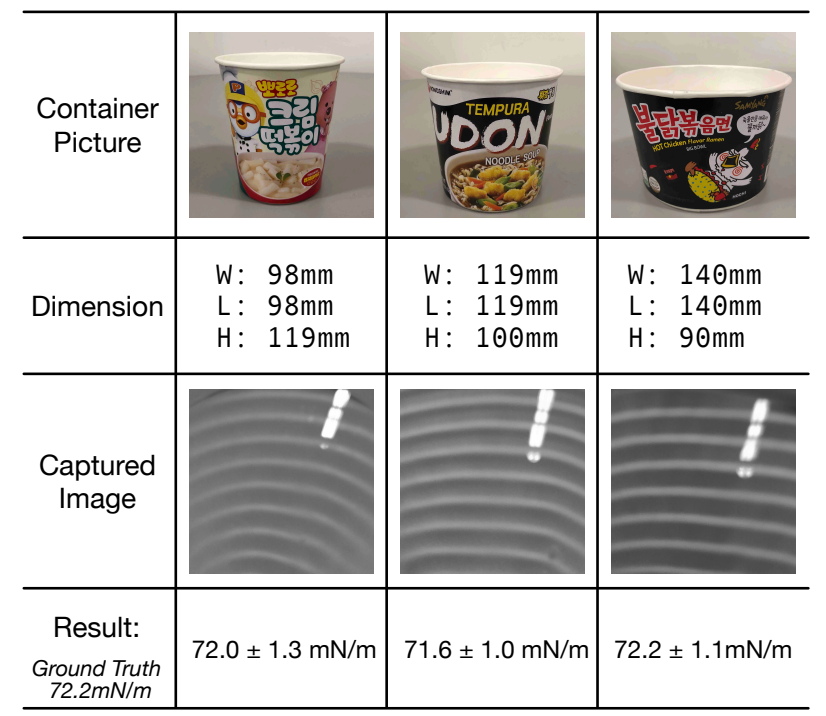

Figure 17: Measuring surface tension with different containers. As the figure indicates, CapCam can provide accurate surface tension measurements with different containers without any modification.

tension values, as well as high variance in the measured wavelength histogram. Further the images of the captured pattern clearly look blurred. When this happens the user should increase or decrease the depth of the liquid until the pattern in the images is not blurred.

\subsection{Impact of Different Containers}

All above experiments are conducted with a standard paper cup because it is a common container easy to find in daily life. But our system is not limited to one container. In Fig. 17, we show the results of experimenting with different containers. We have 
three containers, with different width, length and height. For each container, we add deionized water with depth of $30 \mathrm{~mm}$. We measure the surface tension of the water with CapCam, then compare it with the ground truth measured by the tensiometer, which is $72.2 \mathrm{mN} / \mathrm{m}$. The measurement results show that CapCam provides accurate surface tension measurements with containers of different sizes without any modification.

\section{DISCUSSION AND LIMITATIONS}

(a) Sensitivity: As in past work [11,35], CapCam measures a particular liquid property and uses the measurements to make certain inferences. However, for any of these systems, the inference has to be taken within the measurement context. For example, since CapCam measures surface tension, it is sensitive to bacterial and organic contaminants but cannot sense contamination by heavy metal because they do not change surface tension. Thus, when it infers contamination, the water is certainly impure but the inverse is not necessary true. Similarly, if it detects two liquids to be different, then they are different (assuming no measurement error), but if it cannot differentiate them, they might still be different liquids that have the same surface tension.

It is interesting to note that since CapCam measures a different liquid property than past work, there is an opportunity to combine these techniques for improved performance. In particular, past work measures the liquid electric permittivity, which can be sensed using radio signals that traverse the liquid of interest. In contrast, CapCam measures surface tension which refers to the force that brings the molecules together. These are intrinsically different properties, which combined in a multi-model system are likely to reveal complementary information about the liquid of interest.

(b) Container Specification: CapCam has certain requirements on the container type. First, the container should have a flat bottom. If the bottom is not flat, there will be artifacts in the ring pattern, which affect the measurements. Second, the container should be relatively light so that it vibrates with the vibro-motor. Third, the current system is designed for circular containers. Waves are excited by the vibrating wall and propagate from the edge to the center. Based on the Huygens-Fresnel principle, when the wall is circular, the resulting waves are also circular, hence creating clear rings as described earlier. But if the container is not circular, the wave pattern will be much more complex. Addressing this scenario requires extending the model to account for interaction between waves that traveled different distances, which is left to future work.

(c) Liquid Transparency: CapCam assumes that the liquid is transparent and the pattern at the bottom is visible from the surface. Many liquids are transparent and hence our model directly applies to them. Even when a liquid is not sufficiently transparent, it can be diluted with water and the results can be mapped back to undiluted liquid based on dilution level.

(d) Phone \& Camera Requirements: Capillary waves travel quickly. Hence, when taking images of the waves, it is important to choose a fast shutter speed. New phone models have an API for configuring the camera shutter speed and exposure parameters, and hence our choice of evaluating CapCam on an iPhone X. In our experiments, we set the shutter speed so that the exposure time is $1 / 800$ second. Different phone models may come with different cameras and flashlights, and hence require a different choice for the shutter speed. Note that the automatic exposure configuration will not work because the phone tends to choose a longer exposure time, which causes the wave pattern to be fuzzy. One also needs to measure the resolution of the camera and the vibration frequency of the vibro-motor. These measurements however can be done once for each phone model.

\section{CONCLUSION}

In this paper, we introduce CapCam, the first mobile application that can measure liquid surface tension. It is based on the relationship between surface tension and capillary waves, and it is convenient and accurate. Our evaluation shows that CapCam has an absolute surface tension error of only $0.75 \mathrm{mN} / \mathrm{m}$, and based on measured surface tension, it can successfully measure alcohol concentration and detect water contamination. Our experiments also show that it is capable of accurately tracking the protein level in urine, a key physiological index used in diabetes and kidney disease management. We believe this work can serve as a useful tool, and enables new meaningful applications and interactions.

\section{ACKNOWLEDGEMENTS}

We would like to acknowledge Prof. Gareth McKinley for providing access to the digital tensiometer used for ground truth measurements of surface tension. We thank Deepak Vasisht, Guo Zhang and the members of NETMIT for their insightful discussion and comments. We also thank our shepherd and the anonymous reviewers for their valuable feedback.

\section{REFERENCES}

[1] Apple. 2018. Accelerate Framework. https://developer.apple.com/documentation/ accelerate.

[2] RA Awad, ZMR Hassan, and Wafaa M Salama. 2014. Surface Tension and Foaming Properties as a Simple Index in Relation to Buffalo Milk Adulteration. International Journal of Dairy Science 9 (2014), 106-115.

[3] Tarun Kr Barik, Partha Roy Chaudhuri, Anushree Roy, and Sayan Kar. 2006. Probing liquid surface waves, liquid properties and liquid films with light diffraction. Measurement Science and Technology 17, 6 (2006), 1553.

[4] F Behroozi and A Perkins. 2006. Direct measurement of the dispersion relation of capillary waves by laser interferometry. American journal of physics 74, 11 (2006), 957-961.

[5] Joseph D Berry, Michael J Neeson, Raymond R Dagastine, Derek YC Chan, and Rico F Tabor. 2015. Measurement of surface and interfacial tension using pendant drop tensiometry. Journal of colloid and interface science 454 (2015), 226-237.

[6] Roger D Blandford and Kip S Thorne. 2008. Applications of classical physics. California Institute of Technology.

[7] Edward Bormashenko and Albina Musin. 2009. Revealing of water surface pollution with liquid marbles. Applied Surface Science 255, 12 (2009), 6429-6431.

[8] H Chen, Jesus L Muros-Cobos, and A Amirfazli. 2018. Contact angle measurement with a smartphone. Review of Scientific Instruments 89, 3 (2018), 035117.

[9] H Chen, Jesus L Muros-Cobos, Juan A Holgado-Terriza, and A Amirfazli. 2017. Surface tension measurement with a smartphone using a pendant drop. Colloids and Surfaces A: Physicochemical and Engineering Aspects 533 (2017), 213-217.

[10] Marcelo Cicconet, Vighnesh Birodkar, Mads Lund, Michael Werman, and Davi Geiger. 2017. A convolutional approach to reflection symmetry. Pattern Recognition Letters 95 (2017), 44-50.

[11] Ashutosh Dhekne, Mahanth Gowda, Yixuan Zhao, Haitham Hassanieh, and Romit Roy Choudhury. 2018. LiquID: A Wireless Liquid IDentifier.

[12] Charles J Diskin, Thomas J Stokes, Linda M Dansby, Thomas B Carter, and Lautrec Radcliff. 2000. Surface tension, proteinuria, and the urine bubbles of Hippocrates. The Lancet 355, 9207 (2000), 901-902.

[13] J Drelich, Ch Fang, and CL White. 2002. Measurement of interfacial tension in fluid-fluid systems. Encyclopedia of surface and colloid science 3 (2002), 3158-3163. 
[14] P Lecomte Du Noüy. 1919. A new apparatus for measuring surface tension. The fournal of general physiology 1, 5 (1919), 521

[15] FDS. 2018. Digital Tensiometer - DCAT11. http://www.fdsc.com/wpdev15/ ?portfolio= dca11.

[16] Australian Government. 2018. Microalbumin Level. https://meteor.aihw.gov.au/ content/index.phtml/itemId/270339/.

[17] Nicolas-Alexandre Goy, Zakari Denis, Maxime Lavaud, Adrian Grolleau, Nicolas Dufour, Antoine Deblais, and Ulysse Delabre. 2017. Surface tension measurements with a smartphone. The Physics Teacher 55, 8 (2017), 498-499.

[18] Unsoo Ha, Yunfei Ma, Zexuan Zhong, Tzu-Ming Hsu, and Fadel Adib. 2018 Learning Food Quality and Safety from Wireless Stickers. In Proceedings of the 17th ACM Workshop on Hot Topics in Networks. ACM, 106-112.

[19] FK Hansen and G Rødsrud. 1991. Surface tension by pendant drop: I. A fast standard instrument using computer image analysis. Journal of colloid and interface science 141, 1 (1991), 1-9.

[20] Kyu Keun Kang, Jung Ran Choi, Ji Young Song, Sung Wan Han, So Hyun Park, Woong Sun Yoo, Hwe Won Kim, Dongyoung Lee, Kyoung Hyoub Moon, Myung Hee Lee, et al. 2012. Clinical significance of subjective foamy urine. Chonnam medical journal 48, 3 (2012), 164-168.

[21] Kruss. 2018. Force Tensiometer - K6. https://www.kruss- scientific.com/products/ tensiometers/force-tensiometer-k6/.

[22] Chia-Kai Liang, Li-Wen Chang, and Homer H Chen. 2008. Analysis and compensation of rolling shutter effect. IEEE Transactions on Image Processing (2008).

[23] CE Mogensen. 1984. Microalbuminuria predicts clinical proteinuria and early mortality in maturity-onset diabetes. New England journal of medicine 310, 6 (1984), 356-360.

[24] D Nikolić and Lj Nešić. 2012. Determination of surface tension coefficient of liquids by diffraction of light on capillary waves. European fournal of Physics 33 , 6 (2012), 1677.

[25] Richard J Potton. 2004. Reciprocity in optics. Reports on Progress in Physics 67, 5 (2004), 717

[26] Veronica I Pye and Ruth Patrick. 1983. Ground water contamination in the United States. Science 221, 4612 (1983), 713-718.

[27] Tauhidur Rahman, Alexander T Adams, Perry Schein, Aadhar Jain, David Erickson, and Tanzeem Choudhury. 2016. Nutrilyzer: A Mobile System for Characterizing Liquid Food with Photoacoustic Effect. In SenSys. ACM.

[28] Zhang Shi, Yi Zhang, Mingchao Liu, Dorian AH Hanaor, and Yixiang Gan. 2018 Dynamic contact angle hysteresis in liquid bridges. Colloids and Surfaces A:
Physicochemical and Engineering Aspects 555 (2018), 365-371.

[29] James G Speight et al. 2005. Lange's handbook of chemistry. Vol. 1. McGraw-Hill New York.

[30] MKC Sridhar and C Rami Reddy. 1984. Surface tension of polluted waters and treated wastewater. Environmental Pollution Series B, Chemical and Physical 7, 1 (1984), 49-69.

[31] F Tamm, G Sauer, M Scampicchio, and S Drusch. 2012. Pendant drop tensiometry for the evaluation of the foaming properties of milk-derived proteins. Food Hydrocolloids 27, 2 (2012), 371-377.

[32] Jackson Tan. [n. d.]. Correlation of Urinary Foam with Proteinuria in Patients with Chronic Kidney Disease. ([n. d.])

[33] Gonzalo Vazquez, Estrella Alvarez, and Jose M Navaza. 1995. Surface tension of alcohol + water from 20 to $50^{\circ} \mathrm{C}$. Journal of chemical and engineering data 40,3 (1995), 611-614.

[34] GC Viberti, RJ Jarrett, U Mahmud, RD Hill, A Argyropoulos, and H Keen. 1982. Microalbuminuria as a predictor of clinical nephropathy in insulin-dependent diabetes mellitus. The Lancet 319, 8287 (1982), 1430-1432.

[35] Ju Wang, Jie Xiong, Xiaojiang Chen, Hongbo Jiang, Rajesh Krishna Balan, and Dingyi Fang. 2017. TagScan: Simultaneous target imaging and material identification with commodity RFID devices. In Proceedings of the 23rd Annual International Conference on Mobile Computing and Networking. ACM.

[36] Mengxi Wei, Shuo Huang, Jing Wang, Haihong Li, Hujiang Yang, and Shihong Wang. 2015. The study of liquid surface waves with a smartphone camera and an image recognition algorithm. European fournal of Physics 36, 6 (2015), 065026.

[37] $\mathrm{CH}$ Whitnah. 1959. The surface tension of milk. A review1, 2. Journal of Dairy Science 42, 9 (1959), 1437-1449.

[38] Wikipedia. 2018. Albumin. https://en.wikipedia.org/wiki/Albumin.

[39] Wikipedia. 2018. Surface Tension.

[40] Wikipedia. 2018. Tensiometer (surface tension).

[41] Hui-Shyong Yeo, Gergely Flamich, Patrick Schrempf, David Harris-Birtill, and Aaron Quigley. 2016. Radarcat: Radar categorization for input \& interaction. In Proceedings of the 29th Annual Symposium on User Interface Software and Technology. ACM.

[42] Yuehua Yuan and T Randall Lee. 2013. Contact angle and wetting properties. In Surface science techniques. Springer, 3-34

[43] Feng Zhu, Runcai Miao, Chunlong Xu, and Zanzan Cao. 2007. Measurement of the dispersion relation of capillary waves by laser diffraction. American fournal of Physics 75, 10 (2007), 896-898. 